\title{
The impact of Lotfi Zadeh on my scientific life
}

\author{
Etienne E. Kerre \\ Department of Applied Mathematics, Computer Science and Statistics \\ Ghent University \\ Krijgslaan, 281, building S9 \\ B9000 Gent, Belgium
}

Received: 28 October 2021

Accepted: 20 November 2021

\section{My starting point and pre-fuzzy life}

In 1974, a colleague gave me a bad-smelling photocopy of a paper by Lotfi Zadeh entitled "Fuzzy Sets" [1] that appeared in the Journal "Information and Control" in 1965. At that time, I was looking for a new domain of research. On January 5, 1970, I got the PhD degree in mathematics from Ghent University after a public defense of a thesis on LEED theory, an acronym for Low Energy Electron Diffraction, i.e., a kind of a weak X-ray diffraction with the purpose to study the atomic surface of objects such as crystals with direct applicability to transistors.

A few months earlier, on November 1, 1969, I got an assistantship at my Alma Mater in the newly erected Department of Infinitesimal Analysis where I became responsible for teaching exercises on mathematical analysis to the bachelor students in mathematics. In those days the socalled modern mathematics based on Cantor's set theory became introduced in the university courses. I had to work very hard to modernize our courses on mathematical analysis by introducing the structures of metric spaces, normed linear spaces... to treat the classical concepts such as continuity, convergence, differentiability, integrability. That hard work resulted in the publication of three volumes in the Dutch scientific Reinaert systematic encyclopedia. With respect to research, I no longer could continue in mathematical physics and I had to search for another domain more close to mathematical analysis. That's where Lotfi Zadeh entered my life with his fuzzy sets! 


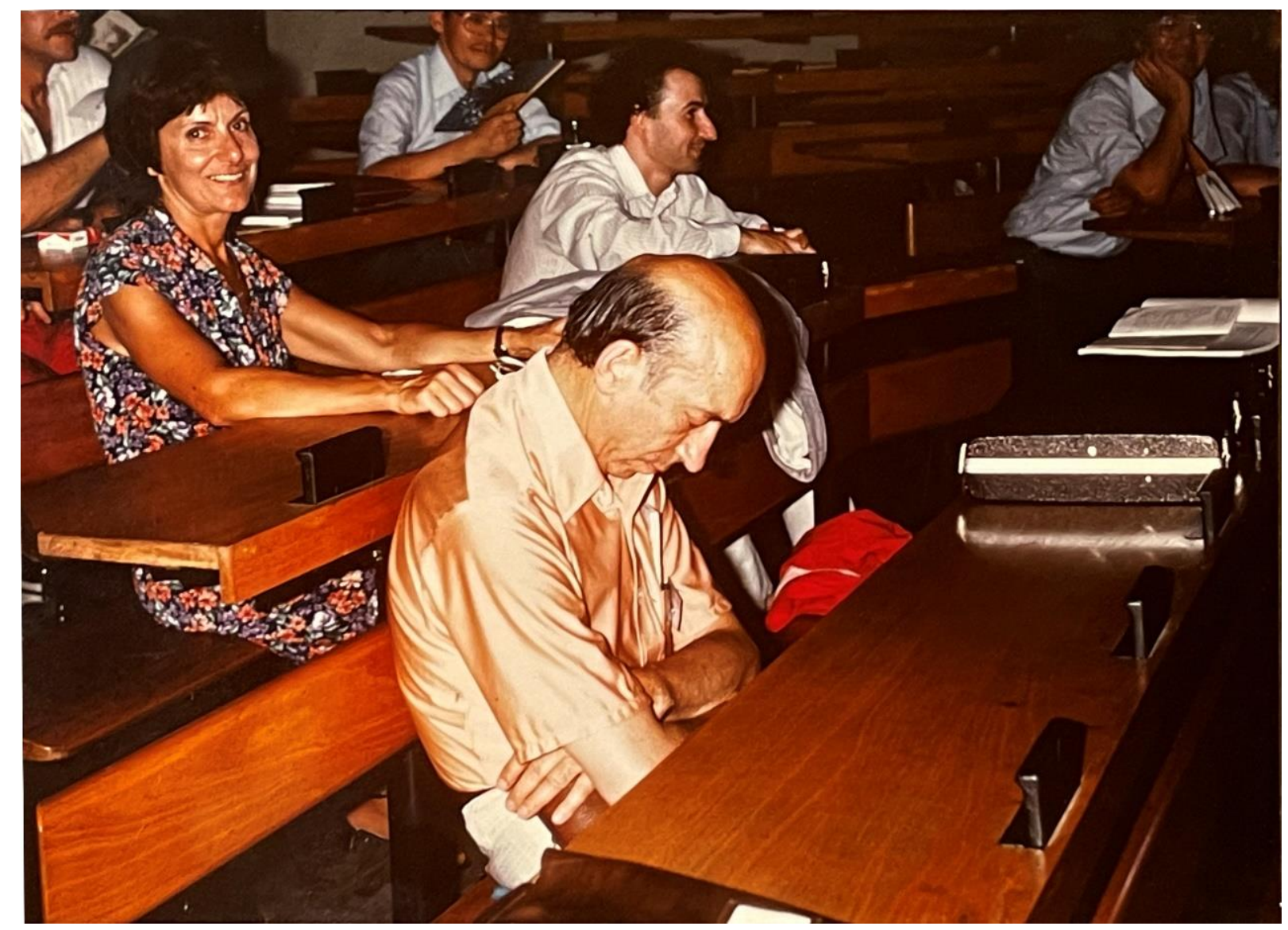

Figure 1. Lotfi Zadeh taking a nap during the first International Fuzzy Set Association Congress in Palma de Mallorca in 1985.

At a first glance his seminal paper has triggered my attention because of the contradictio in terminis: how can a set be fuzzy! I had learned that a set is intuitively defined as a collection of well-defined distinguishable objects! But after reading the introduction I was fully convinced about the great potential of the remarkable concept of a class with more than two possible degrees in fact with an infinite number of membership degrees or grades of membership. I had to admit that in daily life and communication almost all concepts (maybe except purely mathematical concepts such as mapping) are fuzzy and trying to treat them in a binary way leads directly to paradoxes such as the sorites and falakros paradox from the ancient Greeks. For example, in order to decide if a given object belongs to the set of tables one would need big books describing unambiguously all tables which of course would be a never ending process. Nevertheless, the concept of a table never leads to undecidedness in daily life. Also, much later than the old Greeks the famous philosopher Bertrand Russell [2] wrote: "All traditional logic habitually assumes that precise symbols are being employed... it is therefore not applicable to this terrestrial life but only to an imagined celestial existence... logic takes us nearer to heaven than other studies."

Forcing fuzzy concepts into a crisp, precise meaning would be very complicated and artificial. My favorite illustration in this respect is due to Brian Gaines [3].

The sentence "List the YOUNG salesmen who have a GOOD selling record for HOUSEHOLD GOODS in the NORTH of England" is despite the imprecise terms (young, good, household goods, north) perfectly comprehensible. However, in order to make this sentence comprehensive to a classical database system we need a transformation as follows: List salesmen 
under 25 years old who have sold more than 20000 pounds of goods in the categories...to shops in the regions... As a consequence of this precisation, a classical database system will not keep the guy at the age of 26 with a very good selling record, nor the one at the age of 19 with a selling record of 19000 pounds!

Since its introduction at the end of the 19th century by Georg Cantor, set theory has become the language of science in general and of mathematics in particular. The famous mathematician David Hilbert wrote: "Cantor has created a Garden of Eden for the mathematicians and they have decided to stay there forever". Nobody can exaggerate the impact of Zadeh's concept of a fuzzy set: he has immediately enlarged the fundamentals of science and mathematics. Indeed direct fuzzification (i.e. changing the crisp subsets of a universe into the fuzzy subsets and the classical set-theoretic operations by Zadeh's fuzzy set-theoretic operations) has led to the development of fuzzy topology, fuzzy groups, fuzzy vector spaces, fuzzy metric spaces, fuzzy geometry, fuzzy relational calculus...

One of the first domains of direct fuzzification was the introduction of a fuzzy topological space in 1969 by C. L. Chang [4], also my fuzzy starting point in the mid-seventies!

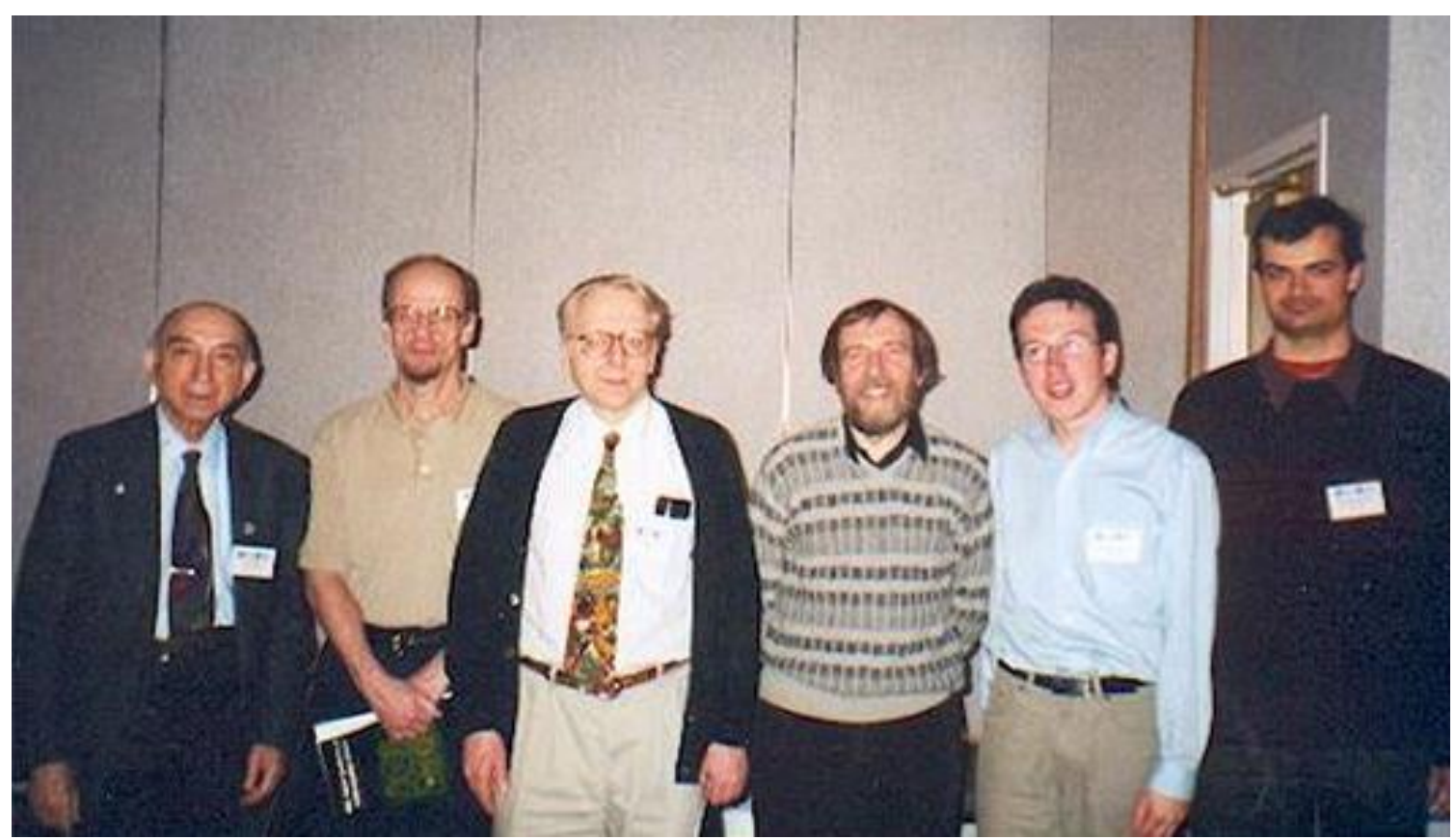

Figure 2. Lotfi Zadeh, Joe Barone, Ladislav Kohout, Etienne Kerre, Chris Cornelis and Glad Deschrijver at the 6th Joint Conference on Information Science in Durham, North-Carolina, USA, in 2002.

\section{My fuzzy research path}

My first steps into the fuzzy world have been in fuzzy topology, a straightforward fuzzification of a classical topology as a class of fuzzy sets that is closed under finite Zadeh intersections and arbitrary Zadeh unions. As every extension during the first fifteen years after Zadeh's seminal paper two objectives were put forward:

- For classical crisp subsets the extension should coincide with the classical concept, i.e., every classical topology should be a special kind of fuzzy topology. 
- Which of the properties of the classical structure are preserved?

My first contribution that I presented at the International Congress of Mathematicians in Helsinki in 1978 concerns the characterization of a fuzzy topology by means of a pre-assigned operation. I could easily prove that a fuzzy topology could be equivalently defined by means of the closed fuzzy sets, the interior operator and the closure operator. However, I failed to prove or disprove the characterization by means of neighborhood systems. Later on, in a series of papers we could completely solve this problem for all kinds of existing neighborhood systems (Ludesher, Kerre, Warren, Pu-Liu, and Mashhour-Ghanim-Kerre). At this conference, I could experience the possible impact of a talk. The ICM congress is held every 4 years with thousands of mathematicians getting 10 minutes to present their work in about 30 parallel sessions. I was lucky to have one of the leading Egyptian topologists in my audience. He was very charmed about the concept of a fuzzy topology and returning to Egypt he started one of the big schools in fuzzy topology! It was a great honor for me to have my first fuzzy topological paper published in the Journal of Mathematical Analysis and its Applications communicated by Lotfi Zadeh in 1980 [5].

A major role in the direct fuzzification of classical mathematical structures has been played by Zadeh's extension principle that appeared already, however a bit hidden, also in his 1965 paper. This principle permits to extend a mapping $f$ from a crisp set $X$ into a crisp set $Y$ to a mapping $F$ from the class of fuzzy sets in $X$ into the class of fuzzy sets in $Y$. Due to this principle it is possible to fuzzify any mathematical structure based on set theory! The most well-known application of this principle is the extension of the arithmetical operations on the real numbers to the calculus of fuzzy numbers. My research team has contributed many papers based on the extension principle. Among them:

- A fuzzification of the binary logic where instead of two truth values (true and false) fuzzy truth values are introduced, i.e., fuzzy sets on the set (true, false).

- Fuzzy relational calculus, especially applied to: medical diagnosis, information retrieval, relational databases, preference relations, multi-criteria decision making, reliability analysis and fuzzy morphology.

For an overview on the different extensions of Zadeh's extension principle we refer to [6].

A second fundamental genuine contribution of Lotfi Zadeh is the introduction of the notion of a linguistic variable, i.e., a variable that takes linguistic values in contrast to a numerical variable that takes numbers as values. A typical example of a linguistic variable is AGE, for example of a person. As a numerical variable it takes values such as: 22 years and 3 months, between 35 and 40 years. As a linguistic value it takes values such as: young, very old, not very old.... In a series of three very long papers Zadeh has explained the basics as well as the enormous amount of possible applications of a linguistic variable.

My research team has contributed a lot to the theory and applications of a linguistic variable, such as: medical diagnosis, database management and the representation of linguistic modifiers. For an overview we refer to [7].

Besides our work related to the extension principle and the linguistic variables, we have done a lot more that resulted in more than 500 publications in international journals and proceedings of international conferences.

I would like to stress that already very early I felt the need to disseminate the existence of such a strong theory with so many potential applications by introducing courses on fuzzy set theory. 


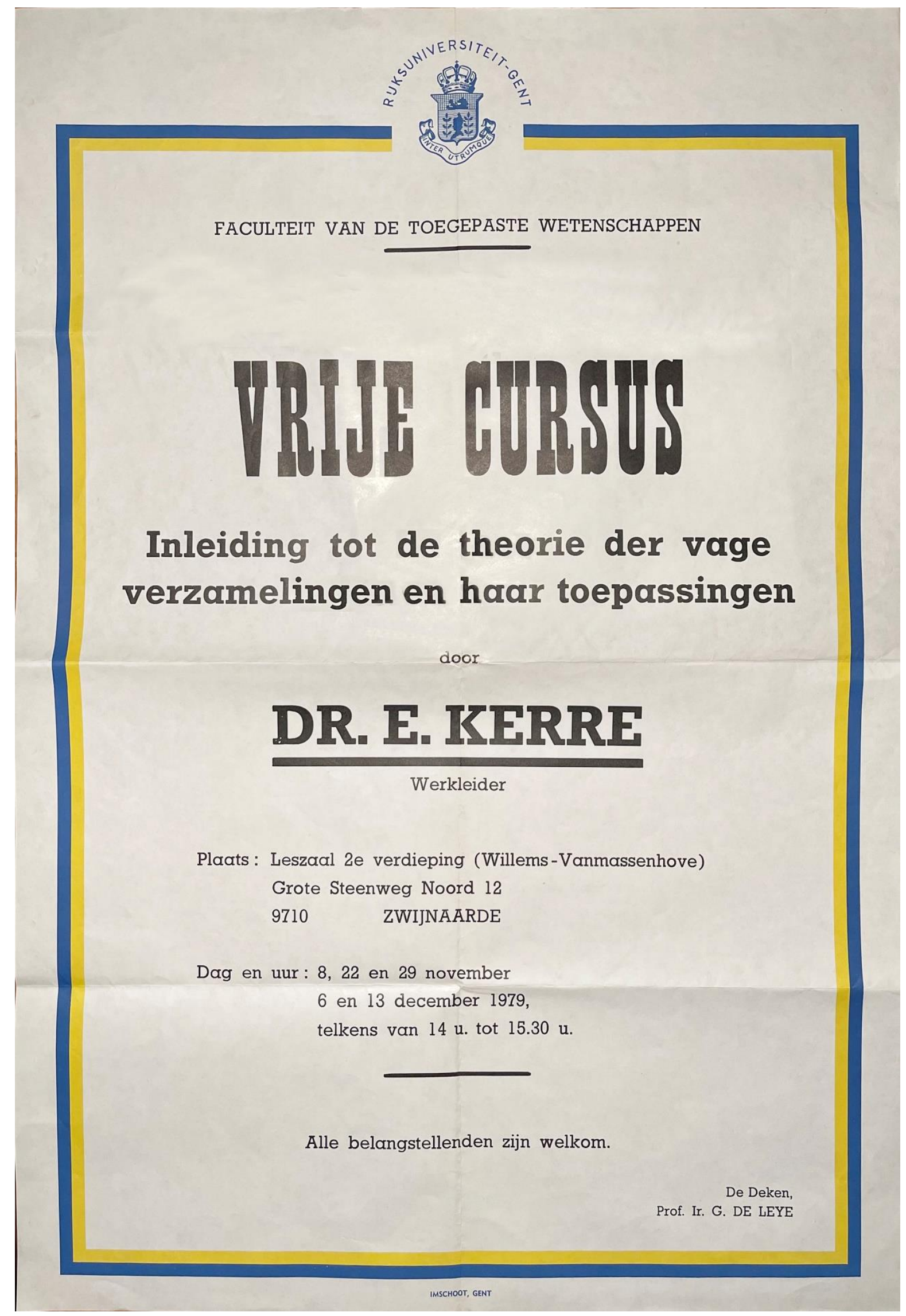

Figure 3. Official announcement of the first optional course on fuzzy set theory (In Dutch: vage verzamelingen) at Ghent university in 1979. 


\section{My fuzzy teaching and coaching}

From the very beginning of my fuzzy life, I was convinced about the need and usefulness of teaching the basic principles of fuzzy set theory. Already in 1979, I accepted the invitation from the Faculty of Engineering of Ghent University to give a short introductory course of 7.5 hours on the theory of fuzzy sets and its applications (cf. the official announcement of this course). About 35 people attended this course (professors, assistants, master students in mathematics). From that time every year I gave an optional course highlighting different topics of fuzzy set theory such as fuzzy algebraic and fuzzy topological structures. Due to the very good response on these optional courses my university decided that in the regular curriculum of the students in mathematics, option computer science, the students could choose a course entitled "Fuzzy sets and their application to computer science" (30 hours per academic year). As a consequence of this course the students in the master mathematics could make their graduate dissertation on fuzzy set theory. In this way, I could attract many clever students.

Besides my courses on Mathematical Analysis to the bachelor Computer Science students, I have taught till my retirement four courses on fuzzy set theory at Ghent University. Here they are:

- Fuzziness and uncertainty modelling (45 hours to students of the third year in computer science and the third year in mathematics).

- Capita selecta from fuzzy set theory (45 hours to students of the fourth year in computer science and the fourth year in mathematics). This course is based on the previous one and it has flexible contents that can be adapted on the current topics of interest.

- Fuzzy logic (30 hours to students of the Postgraduate in Knowledge Technology).

- Fuzzy sets and approximate reasoning (30 hours to students of the Special Graduate in Logics, Methodology and Philosophy of Science).

I have also had the opportunity to teach fuzzy set theory outside my university. Here they are:

- Recent models to handle imprecision and uncertainty in expert systems. Graduate course 496/896 Special Topics in Computer Science. University of Nebraska, Lincoln, USA. (45 hours, Summer 1988).

- Basic principles of fuzzy set theory. Graduate course 496/896 Special Topics in Computer Science. University of Nebraska, Lincoln, USA (45 hours, Summer 1990).

- Application of fuzzy set theory to information retrieval, medical diagnosis, relational databases and expert systems. Graduate course 496/896 Special Topics in Computer Science. University of Nebraska, Lincoln, USA. (45 hours, Summer 1990).

- Fuzzy Technology. Postgraduate course in Postgraduate in Informatics. Alcatel Bell Company, Antwerp, Belgium. (30 hours, Autumn 1993 and Spring 1994). 


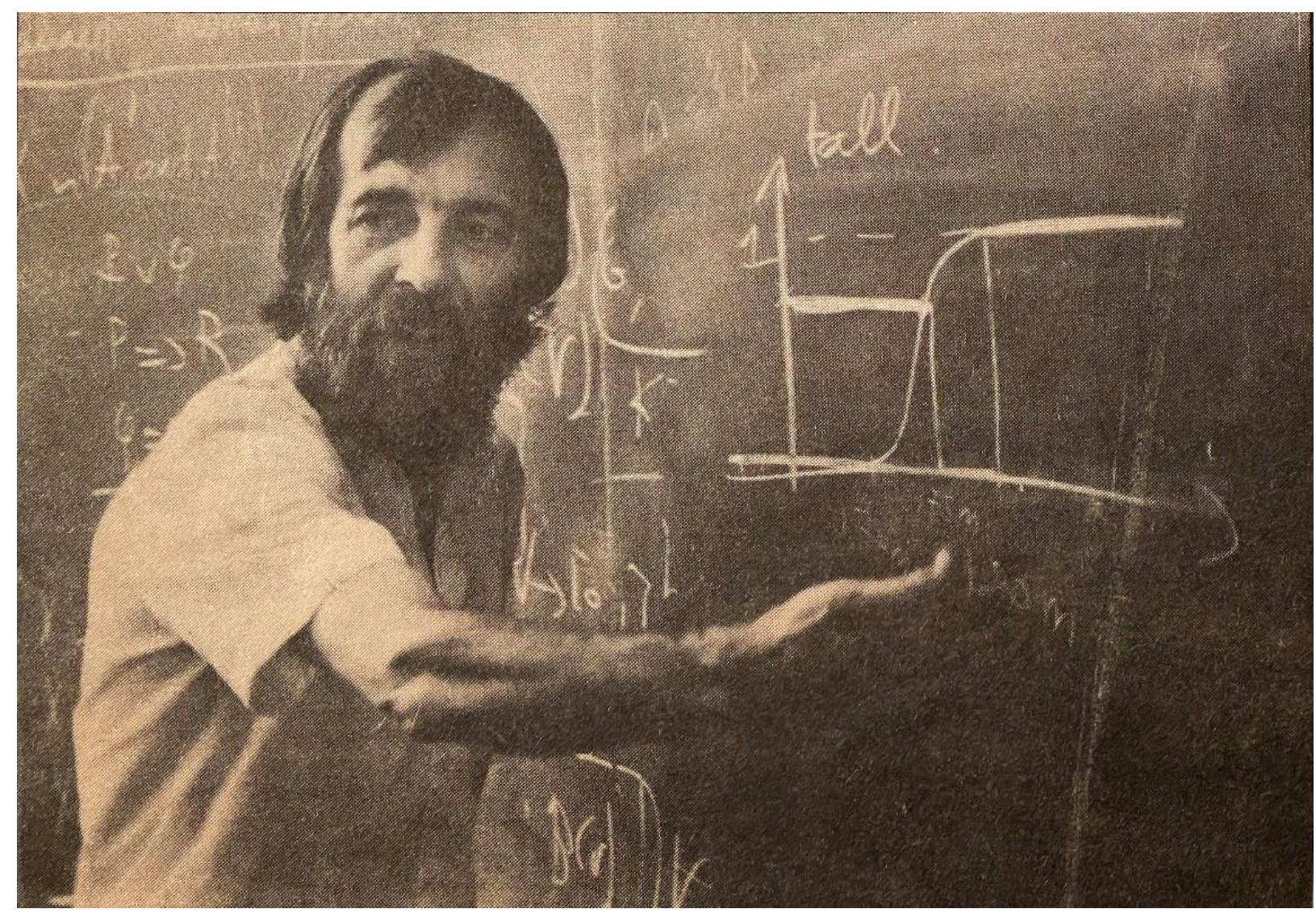

Figure 4. Etienne Kerre teaching fuzzy logic at the University of Nebraska in Lincoln, USA in 1990.

As a result of these teaching efforts on fuzzy set theory, I could convince many excellent students to start research in this beautiful Zadeh world! I guided 21 top students from Ghent University to a PhD degree in fuzzy set theory. Many of them became professors and continued the development of fuzzy sets and related models in their institute (among them: Peter Ottoy, Gert de Cooman, Bernard De Baets, Bartel Van De Walle, Martine De Cock, Chris Cornelis). I also guided $\mathrm{PhD}$ students from abroad who got a grant to do research at Ghent University (among them: Da Ruan, Guoqing Chen, Xuzhu Wang, Yun Shi from China; Mohamed El-Gayyar from Egypt).

\section{Conclusion}

Prof. Lotfi Zadeh has had a tremendous impact on my life during the past 45 years as was illustrated by the huge amount of my publications, my guiding of $30 \mathrm{PhD}$ students and the lecturing of many courses at Ghent University and abroad.

But also I could enjoy many times the kindness of this brilliant researcher. He also took care of my health in stimulating me during breakfast to take some fresh fruit because he observed there was not plenty of it! I will always remember his kind advice: eat your breakfast yourself, share your lunch with your friends and give your dinner to your enemies! Prof. Zadeh was also always very thankful in giving presents after some help or so. I remember a FLINS conference in 2004 he attended in Ghent. I took him with my car very early in the morning to the airport in 
Brussels. Before arriving at the airport he apologized for not having a present for me and all of a sudden he removed his tie and gave it to me saying: please take this tie as a souvenir, it is a brand new one! Prof. Lotfi Zadeh was a scientific giant and a great human being! I am very proud I could extend his ideas!

\section{References}

[1] Zadeh, L. A. (1965). Fuzzy sets. Information \& Control, 8, 338-353.

[2] Russell, B. (1923). Vagueness. Australian Journal of Philosophy, 1, 84-92.

[3] Gaines, B. (1977). Foundations of fuzzy reasoning. In: Fuzzy Automata and Decision Processes (eds. M. Gupta, G. Saridis, B. Gaines), North Holland, New York, 19-75.

[4] Chang, C. L. (1968). Fuzzy topological spaces. Journal of Mathematical Analysis and Applications, 24, 182-190.

[5] Kerre, E. E. (1980). Fuzzy Sierpinski space and its generalizations. Journal of Mathematical Analysis and Applications, 74, 318-324.

[6] Kerre, E. E. (2011). A tribute to Zadeh's extension principle. International Journal of Science and Technology, 18, 593-595.

[7] Kerre, E. E., \& De Cock, M. (1999). Linguistic modifiers: an overview. In: Fuzzy Logic and Soft Computing (eds. G. Chen, M. Ying, K.Y. Cai), Kluwer Academic Publishers, Boston, 69-86. 